\title{
Evolution of Radiation Protection Trends - the Malaysian Perspective
}

\author{
Noriah MOD ALI \\ Secondary Standard Dosimetry Laboratory, Malaysian Nuclear Agency, Bangi, 43000 Kajang, Selangor, Malaysia
}

\begin{abstract}
The growing application of radiation sources demands a better control and efficient radiation protection program, aimed at reducing occupational radiation exposures. In Malaysia, there are continuous efforts and challenges in further promoting radiation protection and safety. A proper, transparent and equitable procedure for competent radiation protection personnel or auditor will require continual development. By having these mechanisms, uniformity of competence and performance can be assured in the promotion of safe behaviour at all levels of the workforce utilising ionizing radiation facilities. Much of the success of occupational radiation protection programmes can be attributed on instituting controls on the radiation work. Individual monitoring service given by Secondary Standard Dosimetry Laboratory, SSDL-Nuclear Malaysia, has undergone a significant evolutionary period during the past few decades. General improvement of protection techniques and technology is expected to continue. Upgrading of the individual dose monitoring service is also highlighted. Reporting program through web based e-SSDL is regarded as a vital step to provide a user-friendly and higher level of confidence on the results generated for occupational dose monitoring of the individual monitoring service. It, thus, enhances the status of the radiation protection framework in the country.
\end{abstract}

KEYWORDS: radiation protection program, individual monitoring service, e-SSDL, safety culture, competence building

\section{Introduction}

Working with radiation, can, in some cases, be dangerous to the safety and health of a worker. Radiation work can be highly hazardous when prevention principles are not applied, where protective measures are not taken and when safe work practices are not adopted due to poor management, or a lack of knowledge, training or assigned accountability and responsibility. A look at regulations from all over the world, one can easily observe that the global approach of the safety at workplaces has already been recommended for many years. People in charge of protection of workers were directed to a more multidisciplinary approach of the work and of their consequences, not only in terms of safety, but also on the "quality" of life of the workers at the workplaces. The ionizing radiation sector has also been involved in such evolution. Since the birth of the ALARA principle, more attention was paid to other factors than the individual dose and the collective dose.

Occupational radiation protection is a team effort. The licensees, regulators, workers, manufacturers and suppliers of radiation sources/equipment, the management, radiation protection officers and other concerned persons are parties to the collective responsibility. Protection primarily depends on the quality of the machine or the source, facility design, workers' capability, and most importantly on the management outlook and efficiency. The words "safety culture" and "quality assurance" are unheard even to most management. Quick turnover of the workers and the responsible persons are also a major hindrance to protection. Regulatory regime is continuously evolving with changing perception and attitude, new scientific knowledge and technological inventions and innovations.

Up to the present, radiation protection is a success story. Although the number of occupationally exposed workers has risen continuously, there has for many years a clear downtrend worldwide, on the doses that workers receive during the course of their work. This trend can be attributed to the widespread practical application of basic radiation protection principles, particularly the principle of optimization of protection, through radiation protection programs and work management. The role of work management is the principal means whereby optimization of protection is implemented. The overall approach to radiological protection is no different from that which applies to the management of the work in general, including all aspects of health and safety. For any place of work where occupational exposure to ionizing radiation is incurred, the operational control of radiation exposures need to be structured, and includes:

(a) a prior radiological evaluation of all aspects of the operation; and 
(b) the establishment of an operational radiological protection program, commensurate the degree of hazard.

The primary responsibility for achieving and maintaining a satisfactory control of radiation exposures rests primarily with the proper management of the operation concerned. Quality Management systems are now an integral part of most businesses, and (radiation protection) self-assessment readily forms a component of such systems, and it requires a continuous process. There is a need to establish a program to measure, review and audit health and safety performance against predetermined standards. This program is known as radiation safety audit and is able to reveal where and when action is needed to make improvements to the systems of controls.

Better protection of workers can be achieved through self-assessment, which is aimed at maintaining regulatory compliance. It is agreed that self-assessment is central to maintaining regulatory compliance, and would usually aim to exceed regulatory requirements.

\section{Status on Radiation Protection}

Radiation protection is one of the factors that need to be addressed to protect the worker's overall health and safety. Better protection of workers is through the emphasis on the human factors of performance rather than technology, and by addressing the common missteps of the management, while suggesting strategies for implementing and strengthening the safety culture. National authorities are necessary to boost the development and implementation of radiological safety and security in the organizations responsible for the uses of radiation sources. The objective of the action programme is to achieve greater awareness by managers and workers on the benefits of a safety culture, with a view to the more widespread adoption of the safety culture approach. A practical guide will also be developed to foster a commitment by managers and workers to the development of a safety culture approach at the enterprise level. These elements are already stressed in the license requirement and again will be taught and reminded in national courses, workshops and seminars to promote radiation safety including internal safety and health audits by workers and managers at the enterprise level.

The introduction of the Atomic Energy Licensing Act, followed by the establishment of the Atomic Energy Licensing Board (AELB) in 1984 were serious initiatives taken by the Malaysian Government to regulate, safeguard and monitor the ionising radiation activities in Malaysia. In addition, AELB is to complement the functions of Malaysian Nuclear Agency (Nuclear Malaysia) that focuses on the application and promoting the peaceful uses of nuclear and related technologies for national development. There has been a significant increase in the industrial applications of radiation sources in Malaysia. In 2008 there were about 2192 workplaces involved with ionizing radiation from 3 categories of job activities, namely medical, industrial and non-destructive testing, NDT (Fig. 1) ${ }^{1)}$. As results, the number of workers in this field is steadily increasing, with around 18,820 radiation workers in 2008 (Fig. 2). Approximately $40.9 \%$ of the total workers are from the industrial, $52 \%$ from medical and $7.1 \%$ from NDT sectors. The medical sector is the oldest user of radiation in Malaysia. Medical use of radiation can generally be split into diagnostic radiology, radiotherapy, nuclear medicine and radiopharmaceutical.

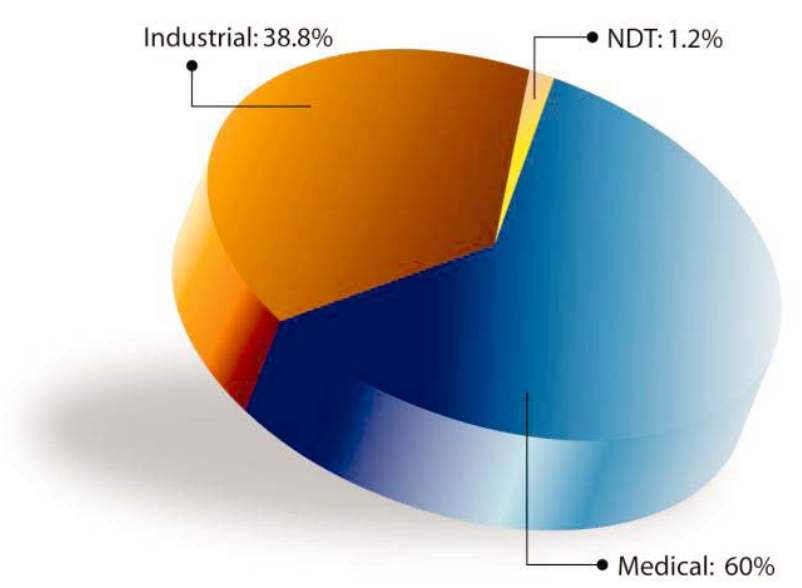

Fig. 1. Portion of workplaces in different sectors involved with ionizing radiation

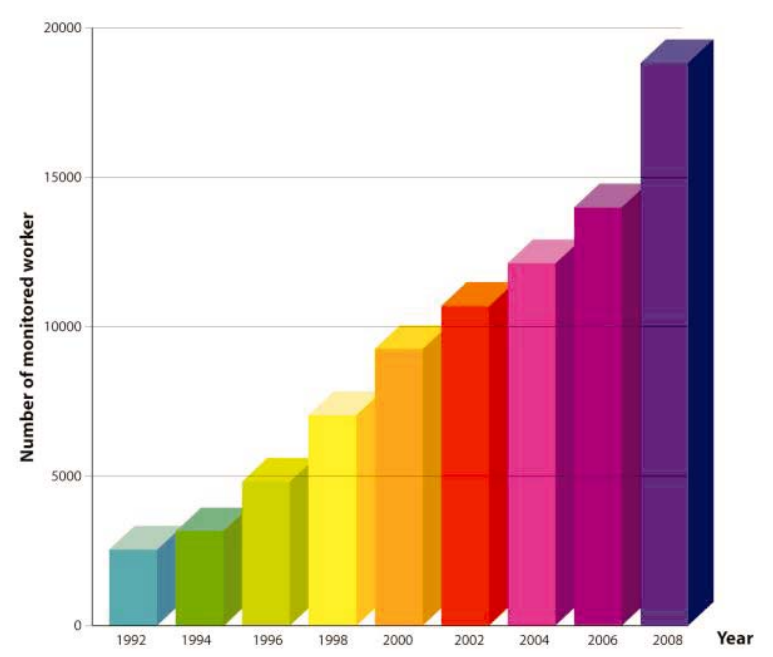

Fig. 2. Increasing trend in number of monitored workers 


\section{Trend of Occupational Dose}

Radiation protection of workers exposed to ionizing radiation in Malaysia is an obligation through national regulations. One of the most important activities in this field covers the individual dosimetry, where film dosimeters (film badge) were used. It was a good solution due to the simplicity of use, the low cost and the assurance of the permanent registration of the dose. The criteria for issuing personal dosimeters is based on the classification of work areas, so all the workers working in controlled areas are assigned to monitoring, while radiation workers working in supervised areas do not require monitoring and are left to their choice on using personal dosimeter. The 25-years period of continuous measurements enables the wide analysis of the annual doses of workers who have been exposed to ionizing radiation.

A national dose registry has been established at SSDL-Nuclear Malaysia, contains radiation doses for all workers and are stored in perpetuity and maintained throughout until the worker cease to be a radiation worker. The individual dose measurement data from various working places can be centrally pooled. Therefore, whenever a monitored person changes work site the individually documented dose histories can still be computed as one lifetime dose history. Obviously, these databases are used as important tools to assess the effectiveness of efforts to maintain doses ALARA. This conforms to the objective of radiation protection program laid through Act 304, 1984, to ensure the safe use of atomic energy in Malaysia.

The routine and systematic collection of data on individual and collective occupational doses categorized by work activity is a key tool to provide information on conditions at the workplace. This information is crucial to identify priority areas for dose reduction and to assess efforts undertaken to maintain ALARA doses. The records on distribution doses achieved through radiation protection control are periodically reviewed. Table 1 shows the trend of occupational exposure for radiation workers in Malaysia from 1992 to 2008. It is interesting to note only $0.5 \%$ of monitored workers received dose greater than $20 \mathrm{mSv}$ in any year, and this was maintained for over a 16-year period. In all cases, where single dose levels are high $(>20 \mathrm{mSv})$, or where accumulative doses approach or exceed the limit (50 mSv.year ), the AELB or Ministry of Health $(\mathrm{MOH})$ will be informed. This will then be followed by an enquiry by the AELB or $\mathrm{MOH}$ and advice on appropriate remedial action.
Table 1. Trend of occupational exposure from 1992 to 2008 in Malaysia

\begin{tabular}{|c|c|c|c|c|}
\multirow{2}{*}{ Year } & \multicolumn{4}{|c|}{ Distribution of workers (\%) over dose interval } \\
\cline { 2 - 6 } & $<0.2 \mathrm{mSV}$ & $0.2-20 \mathrm{mSV}$ & $20.1-50 \mathrm{mSV}$ & $>50 \mathrm{mSV}$ \\
\hline $\mathbf{1 9 9 2}$ & $2042(80.3 \%)$ & $439(17.3 \%)$ & $42(1.6 \%)$ & $20(0.8 \%)$ \\
$\mathbf{1 9 9 4}$ & $2423(76.9 \%)$ & $709(22.5 \%)$ & $16(0.5 \%)$ & $3(0.1 \%)$ \\
$\mathbf{1 9 9 6}$ & $3670(76.2 \%)$ & $1112(23.1 \%)$ & $24(0.5 \%)$ & $10(0.2 \%)$ \\
$\mathbf{1 9 9 8}$ & $5117(72.7 \%)$ & $1809(25.7 \%)$ & $92(1.3 \%)$ & $21(0.1 \%)$ \\
$\mathbf{2 0 0 0}$ & $7808(84.4 \%)$ & $1390(15.0 \%)$ & $44(0.4 \%)$ & $10(0.1 \%)$ \\
$\mathbf{2 0 0 2}$ & $9118(85.2 \%)$ & $1554(14.5 \%)$ & $20(0.2 \%)$ & $4(0.1 \%)$ \\
$\mathbf{2 0 0 4}$ & $10370(85.6 \%)$ & $1721(14.2 \%)$ & $25(0.2 \%)$ & - \\
$\mathbf{2 0 0 6}$ & $12045(86.1 \%)$ & $1903(13.6 \%)$ & $40(0.3 \%)$ & - \\
$\mathbf{2 0 0 8}$ & $16279(86.5 \%)$ & $1883(13.3 \%)$ & $37(0.2 \%)$ & - \\
\hline
\end{tabular}

Comparisons of the total collective dose during the 16 -year period indicated a $15 \%$ decrease while the average dose decreased by $36.6 \%$. The changes are not proportional to the increment of the number of monitored workers, which was about $62 \%$. Industrial radiographers are among the most highly exposed group of workers as compared to others. The nature of their work, the strength of the sources and the energy of radiation used in radiography works contribute to the high exposure. Reduction on the dose received is evident of the implementation of good radiation protection program at the workplace. It indicates proper planning and implementation of the radiation safety program to achieve ALARA doses in the country.

The decrease in average doses may not be sustainable in the face of changes in work requirements. The widening of the scope of application may lead to unwanted events relating to radiation technology. In specific work that involves high routine exposure, safety relies largely on procedures and human performance. Analyses of accidents have identified the major cause as failure to follow procedures, especially with regards to the use of proper dosimeter and personal protective equipment (PPE). Findings from the surveys on several facilities using ionizing radiation showed that internal safety audit is the most common aspect ignored in almost all facilities.

\section{Training in Radiation Safety}

Nuclear Malaysia and AELB strive to keep radiation doses as low as reasonably achievable (ALARA) for workers that use radiation sources in Malaysia. There are also adequate arrangements on education, training and public information and resources for these, as well as appropriate means of 
informing the public, its representatives and the information media on health and safety concerns.

Nuclear Malaysia has been providing training courses on radiological protection for 30 years and has extensive experience in the development of training materials. A wide range of training courses in radiological protection are currently provided by training organizations, both nationally and internationally, and significant effort has been devoted in determining appropriate levels of training, methods of training provision, course content and training infrastructure. The occupational level training courses currently vary from one-day courses for operators of straightforward equipment such as X-ray baggage inspection cabinets, to week-long courses for radiation protection supervisors in a wide range of practices. The number of participants increases each year, and in 2008 around 2845 participants from several sectors, i.e. Radiation Safety and Health (64.5\%), Medical X-ray (16.5\%), NDT (10.1\%) and Environmental Safety and Health $(8.9 \%)$ were trained.

Nuclear Malaysia has pioneered and conducted training for internal auditing of the radiation safety in the past three years for the public and private sectors. The radiation safety audit course is developed with modules describing the content of the audit trainings and the performance of internal audit in organization dealing with nuclear technology. Through this course, radiation protection officers will able to conduct selfaudit in their own premise. This will certainly benefit an organization with ultimate goals of continuously striving for a healthy, accident-free and environmentally sound workplace and community, while providing the technical support needed to meet the national mission.

\section{Competent Radiation Protection Officer}

Efforts have been given on providing a scheme on certified personnel in radiation safety auditing. One of the characteristic functions of the personnel certification body is to conduct an assessment that uses objective criteria to determine if an individual has the required knowledge, skills and abilities to perform a service. It is recognized that such an assessment, if well planned and structured by the certification body, can substantially serve to ensure impartiality of operations and reduce the risk of a conflict of interest, ensuring that an objective certification decision has been reached.

Radiation safety audits are an in-house means of ensuring radiation safety compliance at all times ${ }^{2,3,4)}$. Through self-audit, the performance of the radiation safety can be monitored to check its effectiveness and to achieve stakeholders' satisfaction. The data and findings from the audit can be used as a basis for decision within the organization through adequate analysis. It is useful to describe improvement or deterioration in the system and may give chance for early action to prevent any non-conformity in the system. Opportunities for improvement raised during the audit can be carefully considered and used to improve the implementation of the existing systems in each and every radiation facility in Malaysia whenever appropriate.

\section{RSACB -Certification Body for Radiation Safety Auditor}

In 2008, Nuclear Malaysia initiated efforts on the need to further recognize competence of the radiation protection personnel. Such recognition is required from a competent authority in order for the professionals to be eligible to undertake certain defined radiation protections responsibilities. The process of certification is based on the requirement ISO/IEC 17024:2003 (Conformity assessment - General requirements for bodies operating certification of persons). The scheme will provide international recognition for Radiation Safety Auditor Certification Body (RSACB), which establishes a system for the certification of personnel (known as Radiation Safety Auditor) who are employed to perform radiation safety audit in radiation industries in accordance with Malaysia's own Atomic Energy Act (Act 304) and International Standards. The certified Radiation Safety Auditor may be employed within the radiation facility organization as part of the quality assurance, or by third party inspectorates, inspection bodies, purchasers or consultants.

The scheme is a competency-based personnel certification scheme and was developed with the Atomic Energy Act, Act 304. The scheme has been developed to meet the following key objectives:

(a) To achieve the requirements of the International Standard for personnel certification ISO/IEC 17024:2003;

(b) To ensure that auditors meet or exceed the certification audit, the related competencies defined by RSACB using Act 304, ISO 9001:2008, ISO 19011:2002, OSHA 18001:2005 and ISO/IEC 17021:2004.

(c) To meet the specific requirements of the Certification Body;

(d) To assist the Certification Body in selecting competent auditors; and

(e) To enhance the professional recognition of RSACB certified auditors.

This scheme specifies the qualification and certification of personnel involved in radiation safety auditing in radiation industries in accordance to national and international standards. As a certification body, Nuclear Malaysia will be responsible for the 
certification system, includes all procedures necessary to demonstrate the qualification of an individual to carry out tasks in a specific radiation safety audit method in various industrial sector, leading to certification of competence. The certification would be able to:

(a) Establish and confirm by examination the auditing competency, knowledge and skill of radiation safety auditor to verify the conformity of radiation safety in the industries audited according to the national and international standard;

(b) Provide documented evidence of the radiation safety auditor proficiency in the form of a certificate;

(c) Provide radiation safety auditor a certification that will be recognized and respected by certification bodies, industry, inspection bodies and authorities in Malaysia and internationally; and

(d) Provide a certification system for radiation safety auditor that is transparent, uniform and equitable.

As a certification body, Nuclear Malaysia shall gained accreditation by independent agency - many of which are government sponsored, and in Malaysia it is known as the Department of Standards, Malaysia (Standards Malaysia) (Fig. 3). Standards Malaysia has gained recognition through the acceptance into the Pacific Accreditation Cooperation (PAC), Multilateral Recognition Arrangement (MLA) and acceptance into International Accreditation, Forum (IAF). Accreditation granted by accreditation body members of the IAF Multilateral Recognition Arrangement (MLA), based on regular surveillance to assure the equivalence of their accreditation programs, allows companies and persons with an accredited conformity assessment certificate in one part of the world to have that certificate recognized everywhere else in the world.

The certification is engaged in a continuous review and improvement of auditor competency. By harmonizing the certification process, it will provide a basis to promote a self-check/self-audit culture among radiation practitioners. This will result in a better working situation and confidence in a radiation protection community.

\section{Updating Individual Monitoring Service}

Occupational exposures monitoring in Malaysia is carried out by the Personal Dosimetry Service of the SSDL-Nuclear Malaysia. The service began in 1985, where SSDL-Nuclear Malaysia has been entrusted to issue personal monitoring devices such as film badge

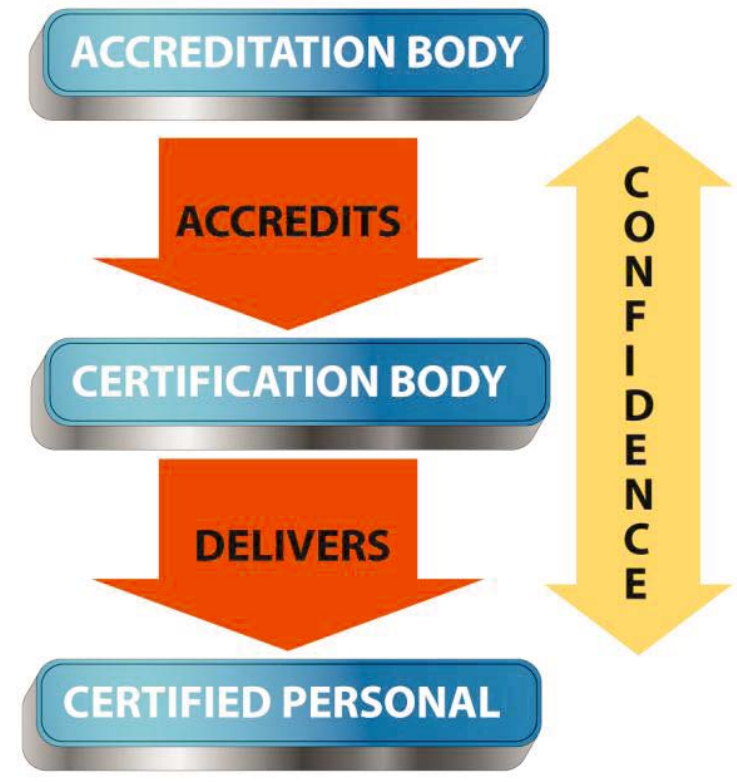

Fig.3. System of Personal Certification

and thermo luminescent dosimeter (TLD) for assessing dose resulting from occupational exposure to ionizing radiation. The main tasks and capabilities of SSDLNuclear Malaysia include the supply and processing of dosimeter systems, as well as evaluation of the dosimeters and issuing of occupational dose reports. The monitoring of individual workers is carried out on monthly basis and dosimeter readings will serve to ensure compliance with legal dose limits and assure employers that radiation exposure in their premises are kept as low as reasonably achievable. Normal processing and reporting turnaround time is within 4 weeks upon receipt of the dosimeters. Same day emergency processing is also available.

With the basic aim of improving accuracy of radiation dosimetry, the SSDL-Nuclear Malaysia demonstrates an evolution in maintaining the performance of the individual dose monitoring services. The major improvement is the development of a better recording and reporting program, which is the key issue for the efficient individual monitoring service. The development and maintenance of proper reporting program is crucial for sustainable confidence of users and regulators on the individual monitoring service.

Beginning in 2008, an interactive open source web based e-SSDL, has been introduced as a suitable platform for easy handling and data access by the customer, operator and the authority. The program is designed to link with full cycle of individual monitoring activities which includes film handling, evaluation, dose report generation, calibration, statistical analysis, inventory, status of company and users (Fig. 4). The system should be able to promptly response on any process of the individual monitoring 
service which can be beneficial to all parties i.e. user, operator and authority. The system is required to address the need to expedite the whole process cycle i.e. supply of dosimeter, receiving, evaluation, readout and generation of a dose report. The system is designed to incorporate a comprehensive tool that covers all aspects of efficient individual monitoring program, in particular:

- Online dosage report.

- Online status of dosimeter supply.

- Monitoring of laboratory efficiency.

- Inventory of dosimeter and other equipment.

- Online search for clients, radiation workers and others.

- Dosimeter calibration and generation of calibration data.

- Integration with barcode system and optical densitometer.

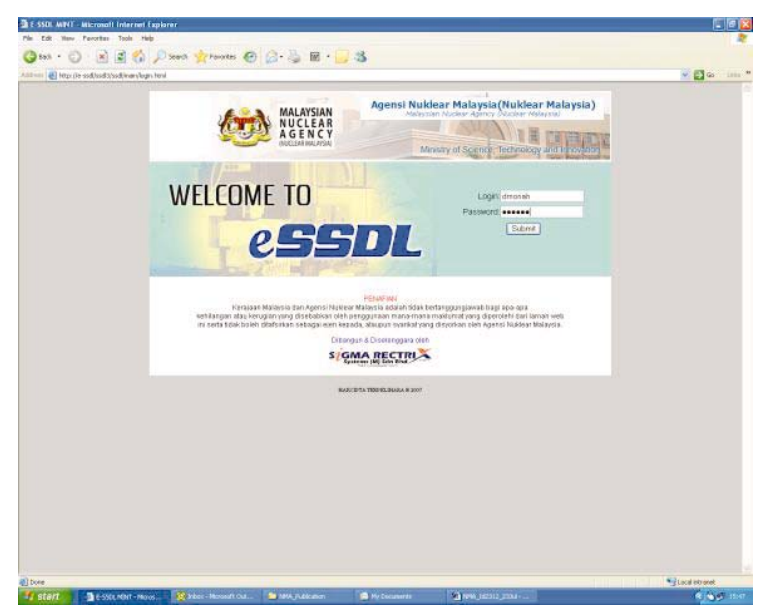

Fig. 4 Entry screen of the e-SSDL

During the first year of implementation, the eSSDL has able to improve the reliability of service to $81.61 \%$, which is better than the SSDL Vb.6 program, by $80.3 \%$ and $76.4 \%$, in 2007 and 2006 , respectively. The efficiency of the service can be improved further as the operator becomes familiar with the system.

\section{Conclusion}

The introduction of Radiation Safety Auditor Certification Body is seen as a competitive product to meet the current and seen future needs in sustainability of radiation safety. By harmonizing the certification process, the standard will provide a basis for international recognition agreements and will promote the global exchange of personnel. It will enable people to expand their career horizons and have their credentials recognized outside of their own company or jurisdiction. As a result, safety culture, which has been a vital element on safety can be improved and utilized to promote changes from good safety performance leading to excellence.

The utilization of a user-friendly and effective occupational exposure evaluation program marks the continual improvement to properly discharge of the responsibilities and efficiently perform the regulatory activities. It will provide a solid foundation to earn strong status in strengthening and maintaining public confidence in the radiation protection framework of the country. In this way, the safety of individual industries and society, in general, can be enhanced for the common good.

\section{References}

1) Noriah Mod Ali, Trends in Occupational Exposure in Malaysia, $11^{\text {th }}$ International Congress of the International radiation protection Association, Madrid, Spain, $23^{\text {rd }}-28^{\text {th }}$ May 2004.

2) Noriah Mod Ali, Challenges in Promoting Radiation Safety Culture, 4th Symposium on Radiation Safety and Detection Technology, 1920 July 2007, Korea.

3) Noriah Mod Ali and K.P.I.K. Kadadunna, Radiation Safety Audit, 12th International Conference on International Radiation Protection Association, Buenos Aires, 19-24 October 2008.

4) Noriah, M.A., Promoting Safety Culture in Radiation Industry through Radiation Audit, IAEA-CN-142/33, Proceedings of an International Conference on Challenges Faced by Technical and Scientific Support Organizations in Enhancing Nuclear Safety, Aix-de-Provence, France, 23-27 April 2007

5) International Nuclear Advisory Group, Key Practical Issues in Strengthening Safety Culture INSAG-15, Vienna, 2002.

6) IAEA Safety Standards, Management Systems for Technical Services in Radiation Safety, DS 315, IAEA, 2005.

7) Radiation Safety Management SystemSpecifications, AELB, Malaysia, 2005.

8) Malaysian Standard, MS ISO/IEC 17024:2003, Conformity Assessment - General Requirements for Bodies Operating Certification of Persons, Department Standard Malaysia, 2003.

9) IAF Guidance on the application of ISO/IEC 17024:2003, Conformity Assessment - General Requirements for Bodies Operating Certification of Persons, International Accreditation Forum Ind. 2004.

10) Noriah M.A., Gaining Competitive Advantage in Personal Dosimetry Through ISO 9001 Certification, Radiat. Prot. Dosimetry, ncl381v1, 2006. 